\title{
Localized amyloid 'tumours' of the lung simulating malignant neoplasms
}

\author{
R. E. COTTON AND J.W. J ACKSON \\ From the Bland Sutton Institute of Pathology, Middlesex Hospital, London, and Harefield \\ Hospital, Middlesex
}

Amyloidosis has been classified by Symmers (1956) into (1) generalized secondary amyloidosis; (2) generalized primary amyloidosis ; and (3) localized amyloidosis.

Involvement of the respiratory tract by amyloid material in the generalized systemic form of the disorder is not as rare as is commonly supposed. In amyloidosis associated with a recognized predisposing disease the incidence is at least $10 \%$ (Dahlin, 1949), and in generalized primary amyloidosis in the absence of any recognized predisposing disease Symmers (1956) found involvement of the lungs in $30 \%$ of 145 cases. In both these groups of cases, which form the majority of all patients with amyloidosis, pulmonary symptoms are usually overshadowed by the severity of changes in other organs, notably the kidneys, spleen, and liver in the secondary disease, and the heart, alimentary tract, liver, spleen, and kidneys in primary amyloidosis. Occasionally, however, diffuse pulmonary involvement, particularly in secondary amyloidosis, may be the dominant clinical feature with death occurring from respiratory insufficiency.

A further, but relatively rare, type of amyloidosis is localized to one organ or anatomical site ; diffuse disease does not develop and there is no known predisposing condition. The commonest sites where such lesions have been reported as occurring are the skin and mucous membranes (mouth, vagina, and conjunctiva), the urinary bladder and urethra, the upper respiratory tract, particularly the larynx, and the lower respiratory tract including lesions of the trachea, bronchi, and lung parenchyma. In the lower respiratory tract, solitary or multiple tumorous masses may form, often with symptoms due to obstruction of the air passages and clinical and radiological appearances which are readily mistaken for primary or secondary neoplasms of the lung.

In a review of localized amyloidosis of the lower respiratory tract, Prowse (1958) was able to find only 16 cases reported in the literature to which he added two that he had personally observed. From the diagnostic and prognostic viewpoints he emphasized the importance of subdividing these cases into the following three groups according to the distribution of the lesions: group 1, multiple nodules of amyloid material confined to the peripheral lung parenchyma; group 2, a solitary tumorous mass of amyloid material affecting a major bronchus; and group 3, amyloid localized to the lower respiratory tract but of a more widespread distribution, involving the mucosa and submucosa of many of the major air passages; this group he calls diffuse tracheo-bronchial amyloidosis. Although cases in all these groups can simulate lung neoplasms, it is in those of groups 1 and 2 that the presence of localized masses visible radiologically is most likely to cause diagnostic confusion.

This paper reports two cases, one in group 1 and one in group 2 , in both of which the diagnosis of a neoplasm, in one case multiple metastases and in the other adenoma or carcinoma of the bronchus, seemed from the clinical and radiological viewpoints almost certain. These cases are reported in order to increase awareness of these unusual manifestations of a disease that appears to show an actual increase in incidence.

\section{CASE REPORTS}

CASE 1 Mrs. L. N., a civil servant aged 57, was referred for investigation after routine miniature chest radiography in April 1962. She had no symptoms. Previous miniature chest radiographs had been clear in 1958 and 1960.

She had had no serious illness or operations, and clinical examination was normal.

Investigations Urine, no abnormality; haemoglobin $90 \%$; white-cell count 6,500/c.mm. ; E.S.R. 13 mm./ hr. (Westergren). Chest radiographs: "There are three rounded opacities in the right chest; appearances suggest secondary deposits. Tomography of this 


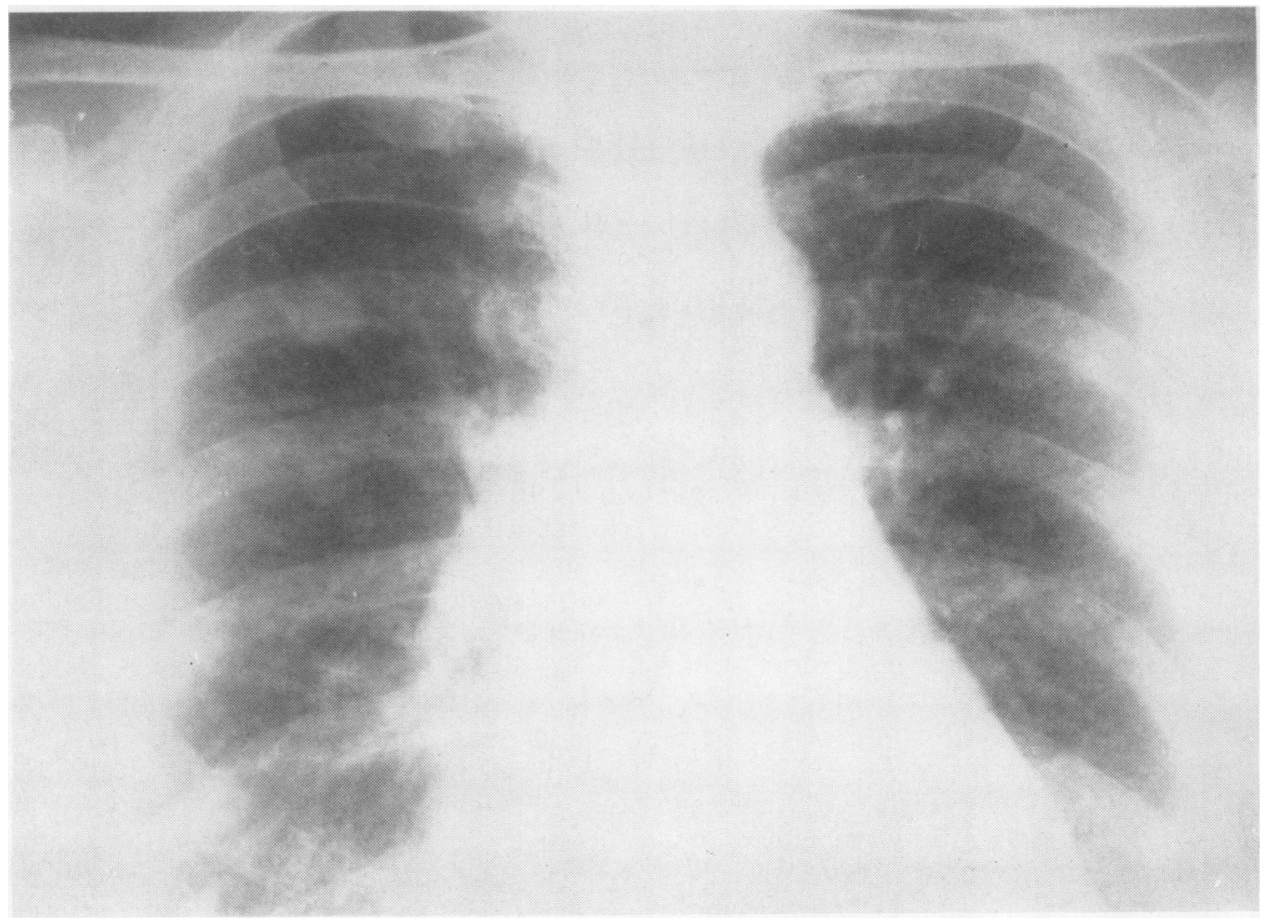

FIG. 1. Case 1. Chest radiograph showing opacities in the right lung.

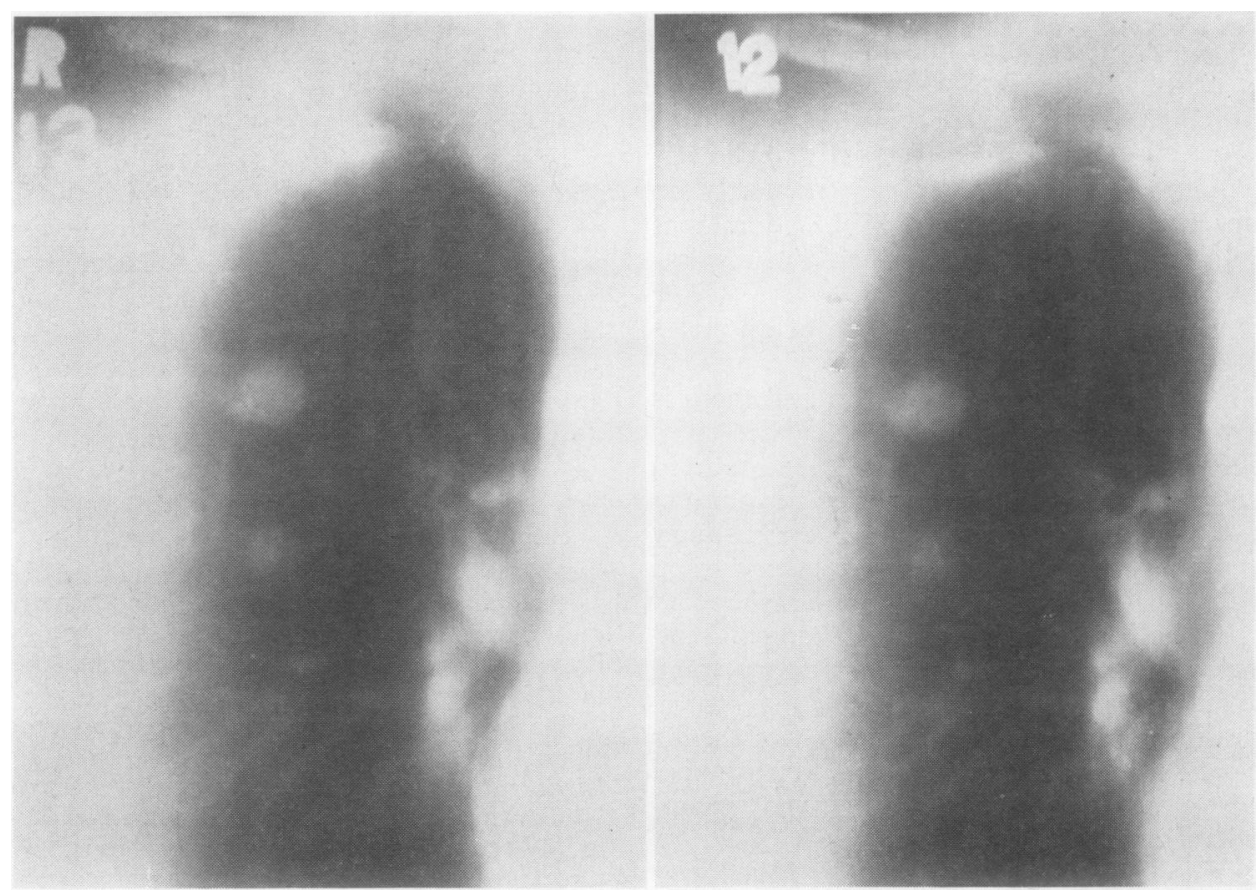

FIG. 2. Case 1. Tomogram showing opacities in the right lung. 
gives no further information. It is noted that the uppermost and largest opacity is not quite homogeneous in density" (Figs. 1 and 2). Barium meal (and follow-through) showed no abnormality. Bronchoscopy was normal.

In the absence of any obvious primary tumour in the breasts, thyroid or pelvis to account for the shadows in the right lung, right thoracotomy and lung biopsy was advised. At operation on 22 August 1962 there was one firm nodule situated centrally in the upper lobe and two smaller nodules in the periphery of the lower lobe, both of which were removed for histological examination.

Pathology The specimen consisted of a portion of pale, firm, glistening, rubbery tissue $\frac{5}{8}$ in. in diameter with a portion of surrounding lung tissue $\frac{3}{8}$ in. in depth, from which it was well demarcated.

Histology revealed that the 'tumour' consisted of concentrically arranged flakes of stranded but otherwise structureless eosinophilic material, uniform in its intensity of staining (Fig. 3). Scattered between the flakes were small foci of plasma cells and lymphocytes (Fig. 4) with rather larger collections of similar cells at the periphery of the lesion. A few residual, metaplastic, cuboidal alveolar cells remained near the

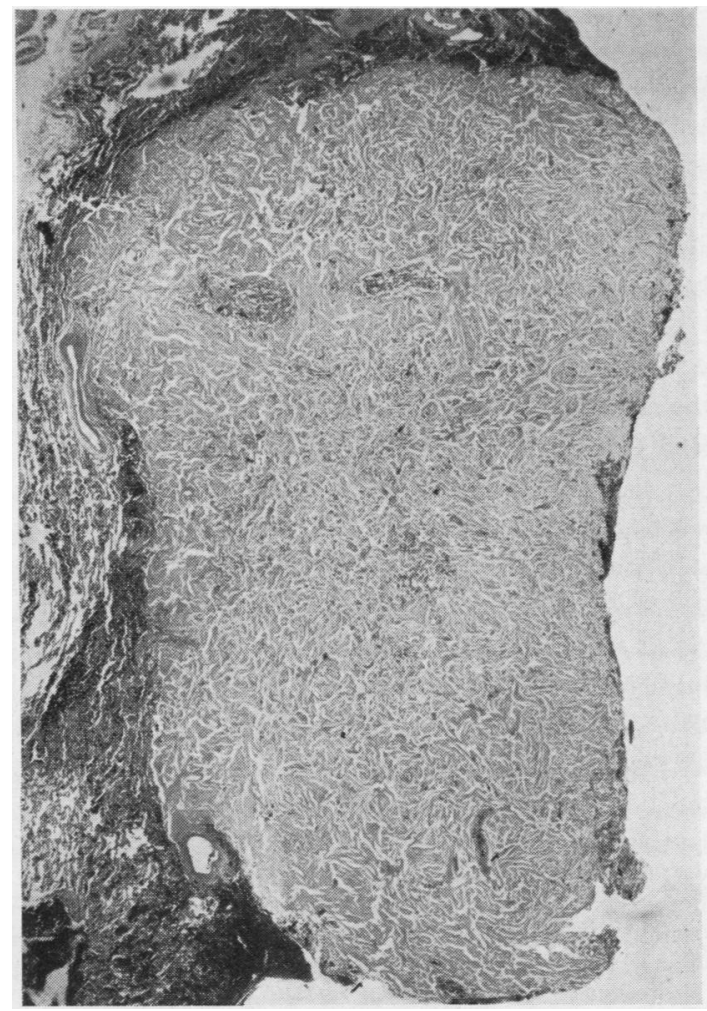

FIG. 3. Case 1. Low-power view of nodule showing the laminated structure and well-circumscribed outline. Haematoxylin and eosin, $x^{\prime} 12$. margin, but otherwise the vascular and alveolar structures had been obliterated. A few giant cells were seen but no bone was found in this lesion. The margins were sharply demarcated, and the surrounding lung tissue appeared entirely normal. No cartilaginous bronchi were present. Sections were stained with Congo red, methyl violet, and thioflavine $T$. There was moderate metachromatic staining with methyl violet and a rather weak positive reaction with Congo red. When examined by ultra-violet light, a section stained with thioflavine $T$. gave the characteristic intense green fluorescence of amyloid material described by Vassar and Culling (1959). This confirmed the identity of the material forming the 'tumour' as amyloid. Fluorescence was limited to the laminated eosinophilic material noted in the haematoxylin and eosin stained section.

The patient has remained well and subsequent radiographs show that the shadow in the right upper lobe has not increased in size.

CASE 2 Mrs. A. J., a housewife aged 58, was seen in June 1961 complaining of tightness in the chest associated with cough and a little phlegm following a recent cold. On two or three mornings she had noticed specks of blood in the sputum. She also

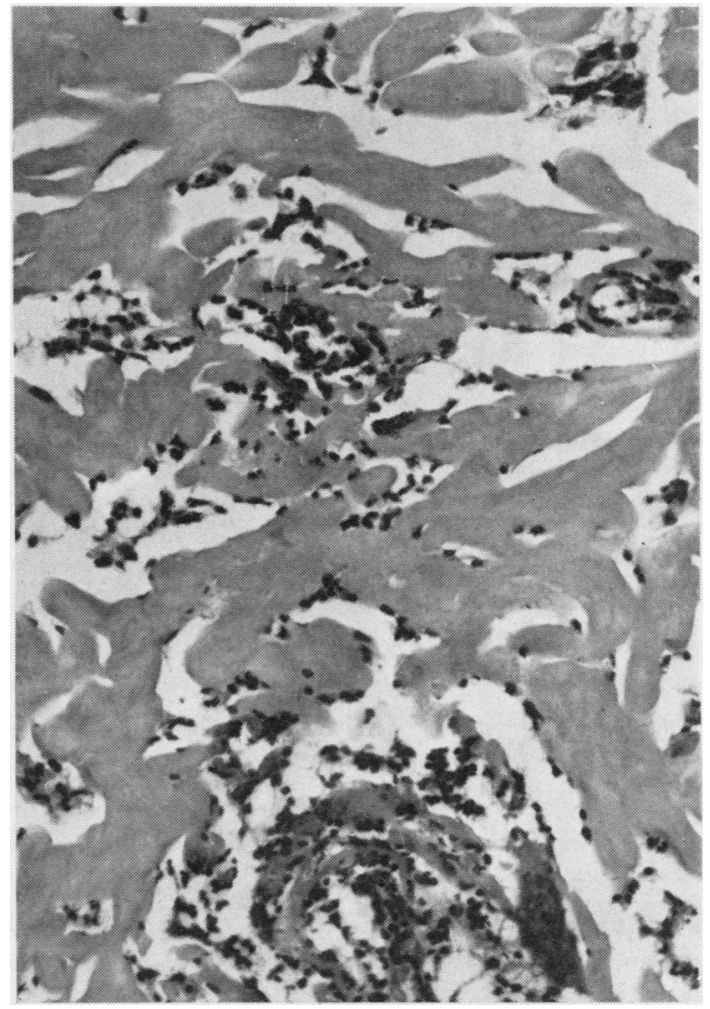

FIG. 4. Case 1. The nodule is composed of strands of structureless amyloid material with small foci of plasma cells and lymphocytes. Haematoxylin and eosin, $\times 160$. 


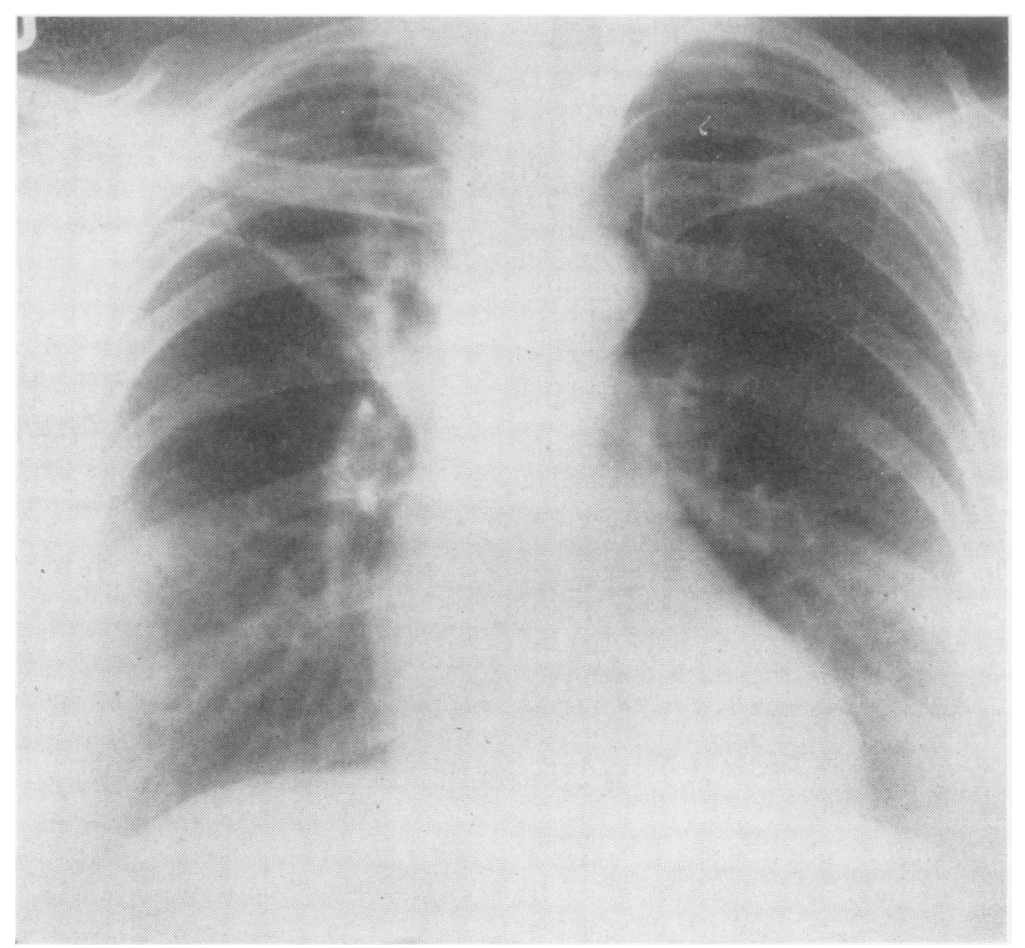

FIG. 5. Case 2. Chest radiograph showing collapse in the right upper lobe.

complained of tiredness and shortness of breath associated with an occasional wheeze.

The previous medical history was not relevant.

On examination she was an obese woman with no anaemia, cyanosis, clubbing or lymph node enlargement.

Chest movements were good. There were harsh breath sounds with a loud inconstant inspiratory wheeze over the right upper lobe. Elsewhere the lungs were clear.

The pulse was regular with normal heart sounds and the blood pressure was $144 / 80 \mathrm{~mm}$. $\mathrm{Hg}$.

The abdomen showed a healed laparotomy scar. The liver and spleen were not palpable.

Investigations Urine, no abnormality ; haemoglobin 91\% ; white-cell count 8,000/c.mm.; E.S.R. $32 \mathrm{~mm} . / \mathrm{hr}$. (Westergren). Spirometry: vital capacity 2.51 . ; M.V.V. 77 1./min.; P.F.R. 300 1./min. Chest radiograph: "The lesser fissure is very high in position, and there is evidence of marked collapse of the anterior segment of the right upper lobe. There is no definite mass visible" (Fig. 5).

Bronchoscopy Some fresh blood was seen in the right main bronchus with tumour in the orifice of the right upper lobe. The left side and remainder of the right side were normal.

Biopsy from the right upper lobe showed considerable deposits of amyloid in the submucosa.
In view of this unusual finding the bronchoscopy. and biopsy were repeated and the findings were confirmed.

Operation At operation on 13 July 1961 there was collapse of the right upper lobe. The tumour in the right upper lobe bronchus was found to encroach on 3 the main bronchus. The hilar lymph nodes were noti enlarged. Right upper lobectomy with sleeve resection 3 of the right main bronchus and end-to-end reanastomosis of the middle and lower lobes to the trachea was carried out.

The post-operative course was uneventful and the follow-up until January 1964 has been satisfactory. $\mathrm{N}$ Pathology Examination of the resected specimen ${ }^{\mathrm{r}}$ showed that the lumina of the right upper lobeN bronchus and its branches were filled with a mass of pale tissue containing areas of obvious bone and $\omega$ cartilage.

Sections stained with haematoxylin and eosine showed laminated masses of homogeneous, structure $-\bar{\Phi}$ less, eosinophilic material in the bronchial wall, pro-ducing gross mucosal thickening with virtual obliteration of the lumen (Fig. 6). This material gave a strongo metachromatic staining reaction with methyl violet (Fig. 7) and stained positively with Congo red. Greend fluorescence after staining with thioflavine $T$. was marked and confirmed the material as amyloid. $\mathrm{Al}$ though predominantly submucosal in distribution? 


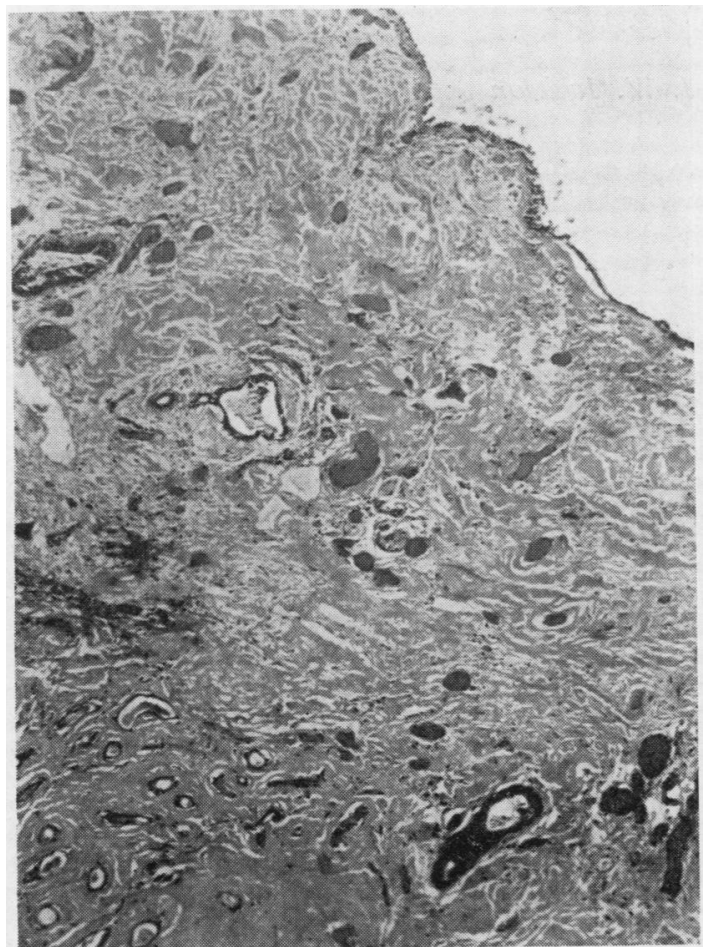

FIG. 6. Case 2. Gross mucosal thickening due to palestaining amyloid material. The surface epithelium remains, but mucous glands are destroyed and only distorted ducts remain. Haematoxylin and eosin, $\times 65$.

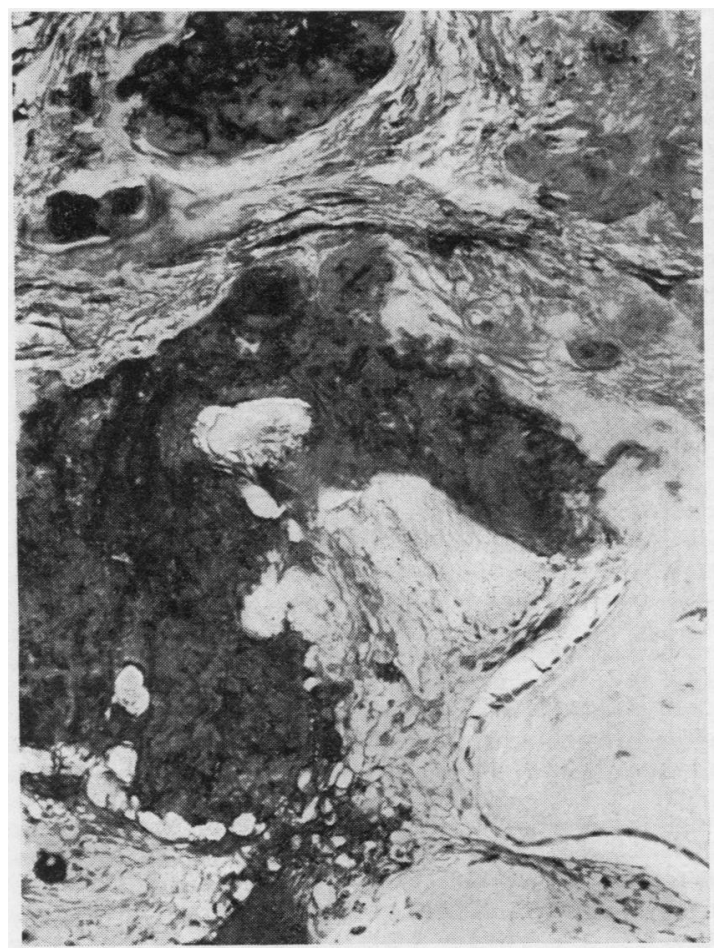

FIG. 7. Case 2. The dark areas are amyloid material stained with methyl violet. Methyl violet, $\times 160$.

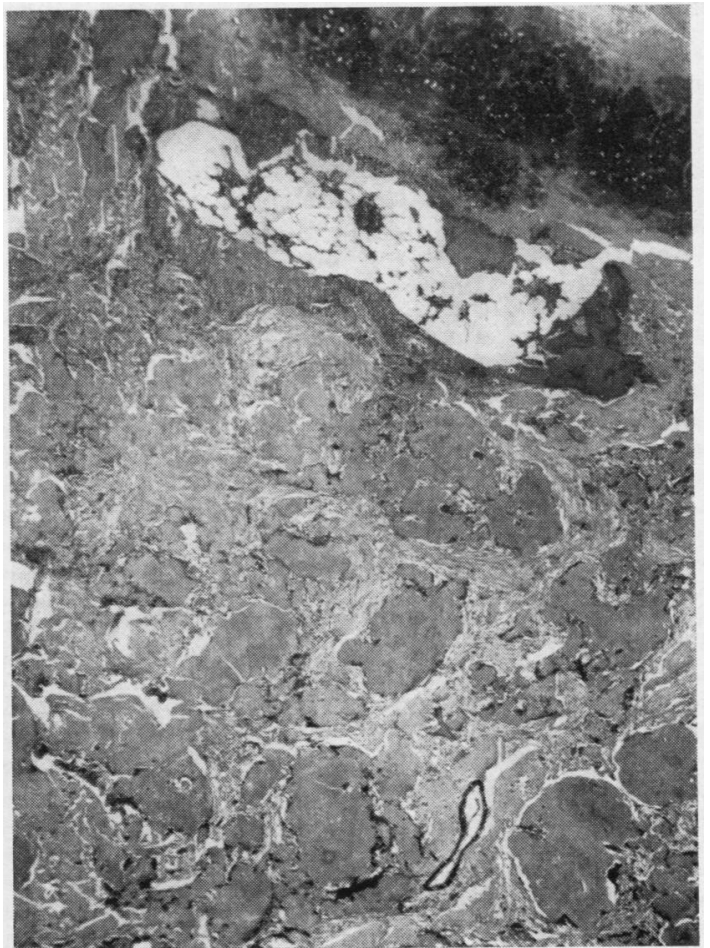

FIG. 8. Case 2. The section shows bronchial cartilage and misses of amyloid material with an area of bone and bone marrow adjacent to the cartilage. Haematoxylin and eosin, $\times 25$.

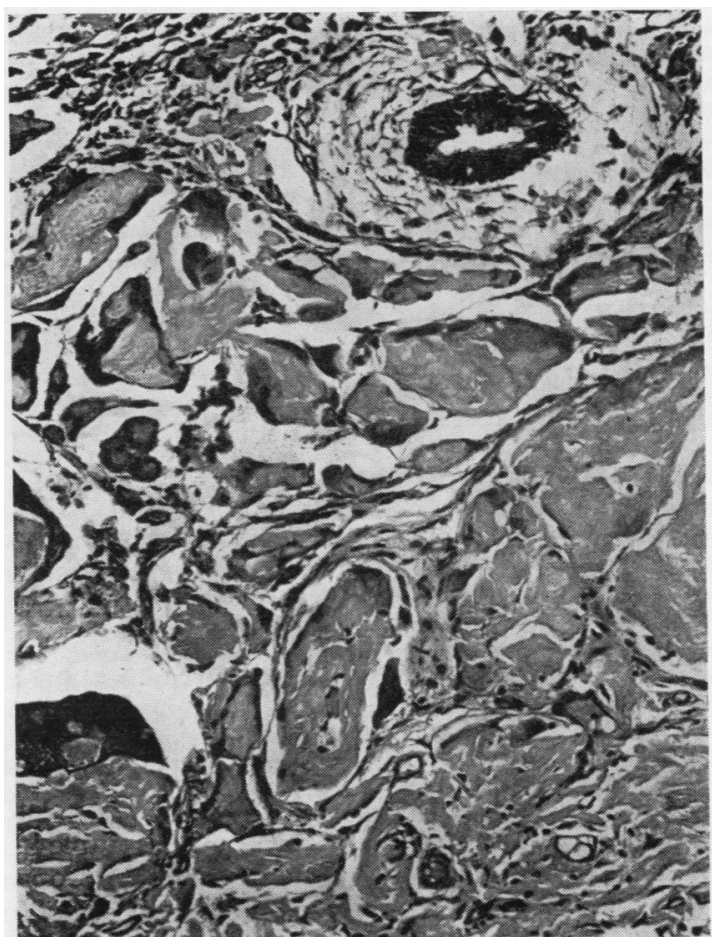

FIG. 9. A mucous gland duct and masses of amyloid material with numerous giant cells. Haematoxylin and eosin, $\times 160$. 
infiltraticn between the cartilaginous rings and into the outer fibrous coat of the bronchus was also present. but surrounding lung tissue was only minimally involved in continuity. The wall of an adjacent large blood vessel was normal as were several tracheobronchial lymph nodes attached to the specimen. A notable feature was the presence within the mass of amyloid material of large amounts of osteoid tissue and cancellous bone with, in areas, the formation of active bone marrow (Fig. 8). Giant cells of foreign body type were numerous in some areas (Fig. 9). Small numbers of plasma cells and lymphocytes were seen in relation to the amyloid material together with occasional large focal collections. Mucous glands and their ducts were largely obliterated by the infiltration but a few dilated and distorted ducts remained (Figs. 6 and 9).

\section{DISCUSSION}

There can be little doubt that both these cases represent unusual manifestations of amyloidosis, apparently confined to the lungs.

MULTIPLE PARENCHYMATOUS TUMOROUS NODULES We have been able to find only four well-documented cases of this distribution in the literature. Cases of multiple parenchymal amyloid nodules have been reported by Meyer (1911), Hallermann (1928), and Lunzenauer (1952) and reviewed by Prowse (1958). Herxheimer's case (1903), accepted by Duke (1959), also had lesions in the larynx and should therefore rightly belong to a different group. All these were incidental necropsy findings in patients who had died from unrelated disease. A further patient, undiagnosed during life, was described by Duke (1959), but symptoms possibly referable to the amyloid disease of the lungs were noted during the terminal illness. The appearances described in these four cases and the distribution of the amyloid material are identical with those of our case 1 , and in all there is no evidence of significant bronchial, tracheal or upper respiratory tract involvement. Evidence of amyloidosis in the liver. spleen, lymph nodes, kidneys, and testes was found in Duke's case (1959) but was slight. Extrapulmonary amyloid was not found in the other cases and there is, as yet, nothing to suggest its presence in this case. The rather flaky, concentric, laminated appearance of this type of lesion is described by all authors and produces an appearance not commonly seen in amyloid deposition in other organs. Groups of plasma cells and lymphocytes related to these localized masses also seem common to all the cases but, in the complete absence of any previous symptoms referable to chest disease and the absence of any necropsy evidence of pre-existing disease, it is not possible to postulate that the nodules are secondary to any localized or indeed generalized predisposing? disorder.

The prognosis in this case is difficult to assess. Three of the four previously reported patients were $\varrho$ asymptomatic and the fourth was 87 years of ages and died from bronchopneumonia, possibly pre- $\vec{\circ}$ disposed to by the pulmonary distortion, the multiplicity of the tumorous masses, and amyloid else- $\vec{\omega}$ where in the body. Comparison with the cases? where the trachea and bronchi are diffusely in- $x$ volved suggests a relatively slow progressive in- $\vec{\omega}$ crease in size, but, if the disease in this casen remains confined to the parenchyma, the short- 0 term prognosis appears good. Regression ofo amyloidosis has occasionally been observed in the secondary type but not recorded with primarys amyloidosis, and experimentally induced amyloid disease in animals has been shown to regress, $\stackrel{\supset}{\supset}$ particularly when associated with the presence of $\vec{\bullet}$ giant cells (Richter, 1954). The length of time that our patient has had amyloid material in the lungs is unlikely to be greater than two years.

SOLITARY BRONCHIAL AMYLOID TUMOURS We have⿳亠丷厂

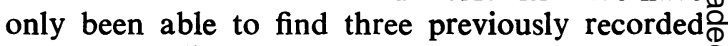
cases of a solitary, or apparently solitary, amyloid $\cong$ deposit in a major bronchus causing obstruction $\overrightarrow{\overrightarrow{0}}$ of the lumen and consequential signs and symp- 3 toms of collapse (Weismann, Clagett, and McDonald, 1947 ; Haynes, Clagett, and McDonald, 1948 ; Schmidt, McDonald, andF Clagett, 1953). All these patients were men aged $\frac{\text { O }}{v}$ 34,35 , and 66 years, whereas our case 2 was ax woman aged 58 . The first two patients had solitary $\frac{\sigma}{3}$ masses in the right lower lobe and right middle lobe bronchi respectively, and both were treated $\mathrm{O}$ by right pneumonectomy with no evidence of recurrence in the lung or elsewhere seven years? and six years later. The diagnosis in both patients was made from the pathological appearances of the operation specimens, the resections having $N$ been performed with a presumptive diagnosis of ${ }_{N}$ carcinoma of the bronchus. The patient of Schmidt 0 et al. (1953) had a carinal lesion involving both $\omega$ main bronchi with a large mass visible on tomography. Biopsy at bronchoscopy showed amyloid material and, since surgical resection was not $ळ$ technically feasible, he was treated by radiotherapy? after bronchoscopic curettage to increase the size $\frac{0}{0}$ of the airways. Four years later this patient had $\underset{\mathbb{D}}{\stackrel{O}{*}}$ a brassy cough but was otherwise well.

The present case had a localized mass, obstruct $-\stackrel{\mathbb{Q}}{\stackrel{\Omega}{Q}}$ ing the right upper lobe bronchus, removed by sleeve resection. There is no evidence of amyloid- 8 
osis elsewhere, and rather more than two years later there is no evidence of a recurrence. Both the short-term and long-term prognoses therefore appear to be favourable.

This seems to be a true example of a local amyloid 'tumour' of the bronchus, and its features are similar to the few examples previously described. The presence of bone and bone marrow in the amyloid material seems usual in this situation, although it is not a common feature of amyloidosis in other parts of the body.

It is often stated that localized amyloid tumours show atypical staining reactions or may fail to stain with the usual histochemical techniques for amyloid material. Consequently some authors have used the term ' para-amyloid ' in such instances, inferring that the material was of different composition. In all the tumorous lung lesions reviewed and in the two cases reported, however, one or more of the classical staining reactions was positive. Fluorescence of amyloid stained with thioflavine $T$. has been found in routine practice to be a most sensitive diagnostic test.

\section{SUMMARY}

Two cases in which the clinical and radiological appearances strongly suggested neoplastic disease in the lungs are described. Both were found to have amyloid disease apparently confined to the lungs. The literature of localized amyloidosis of the lungs is briefly reviewed.

\section{REFERENCES}

Dahlin, D. C. (1949). Secondary amyloidosis. Ann. intern. Med., 31, 105.

Duke, M. (1959). Tumoral amyloidosis of the lungs. Arch. Path. (Chicago), 67, 110.

Hallermann, W. (1928), t'ber eigentümliche Herde in den Lungen, die dem lokalen tumorförmigen Amyloid nahestehen. Frankfurt Z. Path., 36, 471.

Haynes, A. L., Clagett, O. T., and McDonald, J. R. (1948). Tumorforming amyloidosis of the lung. Surgery, 24, 120.

Herxheimer, G. (1903). Über multiple Amyloidtumoren des Kehlkopfs und der Lunge. Virchows Arch. path. Anat., 174, 130.

Lunzenauer, K. (1952). Uber Amyloid-“tumoren" der Lungen. Frankfurt Z. Path., 63, 519.

Meyer, O. (1911). Über lokales tumorartiges Amyloid in den Lungen. Ibid., 8, 304 .

Prowse, C. B. (1958). Amyloidosis of the lower respiratory tract. Thorax, 13, 308.

Richter, G. W. (1954). The resorption of amyloid under experimental conditions. Amer. J. Path., 3,, 239.

Schmidt, H. W. McDonald, J. R. and Clagett, O. T. (1953). Amyloid tumors of the lower part of the respiratory tract and mediastinum. Ann. Otol. (St. Louis), 62, 880.

Symmers, W. St. C. (1956). Primary amyloidosis: a review. J. clin. Path., 9, 187.

Vassar, P. S., and Culling, C. F. A. (1959). Fluorescent stains, with special reference to amyloid and connective tissues. Arch. Path. (Chicago), 68, 487.

Weismann, R. E., Clagett, O. T., and McDonald, J. R. (1947). Amyloid disease of the lung treated by pneumonectomy. $J$. thorac. Surg., 16, 269. 